ESAIM: M2AN

Vol. 40, No 2, 2006, pp. 393-412

DOI: $10.1051 / \mathrm{m} 2 \mathrm{an}: 2006018$
ESAIM: Mathematical Modelling and Numerical Analysis

www.edpsciences.org/m2an

\title{
A VISCOSITY SOLUTION METHOD FOR SHAPE-FROM-SHADING WITHOUT IMAGE BOUNDARY DATA
}

\author{
Emmanuel Prados ${ }^{1}$, Fabio Camilli $^{2}$ and Olivier Faugeras ${ }^{3}$
}

\begin{abstract}
In this paper we propose a solution of the Lambertian shape-from-shading (SFS) problem by designing a new mathematical framework based on the notion of viscosity solution. The power of our approach is twofolds: (1) it defines a notion of weak solutions (in the viscosity sense) which does not necessarily require boundary data. Moreover, it allows to characterize the viscosity solutions by their "minimums"; and (2) it unifies the works of [Rouy and Tourin, SIAM J. Numer. Anal. 29 (1992) 867-884], [Lions et al., Numer. Math. 64 (1993) 323-353], [Falcone and Sagona, Lect. Notes Math. 1310 (1997) 596-603], [Prados et al., Proc. 7th Eur. Conf. Computer Vision 2351 (2002) 790-804; Prados and Faugeras, IEEE Comput. Soc. Press 2 (2003) 826-831], based on the notion of viscosity solutions and the work of [Dupuis and Oliensis, Ann. Appl. Probab. 4 (1994) 287-346] dealing with classical solutions.
\end{abstract}

Mathematics Subject Classification. 35D99, 62H35, 65N06, 65N12, 68T45.

Received: July 21, 2005.

\section{INTRODUCTION}

Shape-from-shading (SFS) is the problem of recovering the three dimensional shape of a surface from the brightness of a black and white image of it. A PDE approach to the shape-from-shading problem leads to the study of Hamilton-Jacobi equations of eikonal type, i.e. equations of the form:

$$
H(x, \nabla u(x))=0, \quad \forall x \in \Omega,
$$

where $H$ is continuous and convex, coercive with respect to $\nabla u$ and $\Omega$ is an open subset of $\mathbb{R}^{2}$ (see for examples $[13,22]$ ). A classical solution (i.e. in pointwise sense) to these equations in general does not exist, so it is necessary to consider solutions in a weak sense. In this framework the most reliable type of solution is the Crandall-Lions viscosity solution $[10,17]$. The theory of viscosity solutions was firstly applied to the SFS problem by Lions, Rouy and Tourin $[18,25]$ in the 90 s.

The work of Lions, Rouy and Tourin is based upon the notion of continuous viscosity solution. In order to characterize a solution, they complement their SFS equations with some boundary constraints, more precisely

\footnotetext{
Keywords and phrases. Shape-from-shading, boundary data, unification of SFS theories, singular viscosity solutions, states constraints.

1 Perception Team, INRIA Rhône-Alpes, France. Emmanuel.Prados@inrialpes.fr

2 Dipartimento di Matematica Pura e Applicata, Università dell'Aquila, Italy. camilli@ing.univaq.it

3 Odyssée Lab., INRIA Sophia Antipolis, France. Olivier.Faugeras@sophia.inria.fr
}

(C) EDP Sciences, SMAI 2006 
Dirichlet boundary conditions. Even if the viscosity solution theory provides general existence, uniqueness and stability results for the class of Hamilton-Jacobi equations arising in SFS, its application is hampered by two types of difficulties.

The first one is the compatibility condition required for the boundary data [17]. If this condition is not satisfied, the boundary data is not assumed in pointwise sense and it has to be interpreted in a weak (viscosity) sense. Moreover, in practice, with real images these data are generally not known or known only partially and can contain errors.

For solving the compatibility condition problem the concept of continuous viscosity solution was extended to the one of discontinuous viscosity solution [4,14]. In [23], this notion was applied to the SFS problem, in particular for studying convergence of approximation schemes when the compatibility condition on the boundary data is not satisfied.

The second difficulty is due to the presence of a set of degeneration for the Hamilton-Jacobi equation. It is well known that Hamilton-Jacobi equations of eikonal type admit a regular subsolution which plays a key role in the uniqueness. If this subsolution fails to be strict even at a single point, then in general uniqueness of the viscosity solution fails. Following [17], this degeneration is well understood: all the viscosity solutions varies between a minimal and a maximal viscosity solution and a solution is univocally characterized by its values on the set of degeneration. In the SFS case, the set of degeneration coincides with the set of points where the light intensity is maximal, thus this difficulty is general and applies to all the SFS equations considered in literature. For computing a solution, Rouy and Tourin [25] propose then to specify the height of the solution at all the singular points. Nevertheless, in general in practice we don't have this knowledge. Also, it is important to minimize the amount of required data.

To circumvent this problem, in [15] for the case of a singular point of degeneration and in $[6,7]$ in the general case, the definition of viscosity solution was modified to the one of singular viscosity solution. The basic idea is to modify the definition of viscosity solution in such a way to recover the existence of a strict subsolution for a modified Hamilton-Jacobi equation and, by this fact, to obtain a characterization of the maximal (minimal) solution. In the previously mentioned papers the singular solution considered is an extension of the continuous viscosity solution with a pointwise boundary condition, while in this paper we consider the corresponding extension for the discontinuous viscosity solution with viscosity boundary conditions.

From the point of view of SFS problem such an extension to the discontinuous viscosity solutions has some significant advantages. Firstly it allows to impose the height of the solution at the singular points when the compatibility condition is satisfied and if necessary it allows to characterize the maximal solution (i.e. the maximal surface admitting a given image brightness function). In particular we can impose the boundary datum where we know it and send to infinity (i.e. impose a state constraints) where we don't know it. Secondly it allows to reconciliate the viscosity solution approach with an alternative approach proposed by Dupuis and Oliensis $[11,20]$, which characterizes the solution by its values at the minimum points with no boundary data. Moreover since this approach is an extension of the concepts of continuous and discontinuous viscosity solution, it unifies in a unique mathematical framework the work of Lions et al. [18], Rouy and Tourin [25], Falcone and Sagona [12], Prados et al. [21,23], based on the notion of viscosity solutions.

In other respects the proposed notion enjoys of another noteworthy property: it maintains the same stability properties as the previous ones. Also we prove in Section 4 a general stability theorem. This result allows in particular to show in this paper (see also [24]) that the solution we consider is stable respect to various perturbation of the SFS problem, for example respect to errors in the SFS parameters (image brightness $I$, focal length $f$, source direction $\mathbf{L}$, etc.) or respect to the approximation via finite differences methods.

The paper is organized as follows. In Section 1, we write a general model of shape-from-shading equation including the more common equations used in literature. In Section 3 we introduce the notion of singular discontinuous viscosity solution. Section 4 is devoted to existence and uniqueness of the previous notion of solution. In Section 5 we study the important issue of the stability and in the following section we apply the result to the study of various perturbations of the shape-from-shading problem. In Section 7 we study some 
additional properties of the solution and we show that it can be characterized by the values at the "minimum" points. Finally in Section 7 we present some numerical results obtained applying the previous model.

\section{HAMiltonians FOR THE LAMBERTIAN SFS PROBlem}

There are various Hamiltonians considered in SFS literature depending on the modeling adopted. In this section we recall some possible formulations of the SFS problem. If $\Omega$ is the image domain (an open subset of $\mathbb{R}^{2}$ ), the surface $\mathbf{S}$ we want to reconstruct can be explicitly parameterized by

$$
\mathbf{S}=\{S(x) ; \quad x \in \bar{\Omega}\}
$$

where $S: \bar{\Omega} \rightarrow \mathbb{R}^{3}$. Assuming that the scene is Lambertian and illuminated by a single light source [13], then the image brightness $I(x)$ of a point $x$ in $\Omega$ verifies

$$
I(x)=\frac{\mathbf{n}(S(x))}{|\mathbf{n}(S(x))|} \cdot \mathbf{L}(S(x))=\cos (\mathbf{n}(S(x)), \mathbf{L}(S(x))),
$$

where $\mathbf{n}$ is a normal vector to the surface (pointing upward the surface) and $\mathbf{L}$ is the unit vector describing the light source direction.

\subsection{Light source at infinity}

When the light source is located at infinity, $\mathbf{L}$ is uniform. We set $\mathbf{L}=(\alpha, \beta, \gamma)$ and $\mathbf{l}=(\alpha, \beta)(\gamma>0)$.

"Orthographic SFS": here, we assume that the camera performs an orthographic projection of the scene. This is the traditional setup for the SFS problem. Choosing $S(x)=(x, u(x))$, the SFS problem corresponds to find a function $u: \bar{\Omega} \longrightarrow \mathbb{R}$ satisfying the following equation:

$$
I(x)=(-\nabla u(x) \cdot \mathbf{l}+\gamma) / \sqrt{1+|\nabla u(x)|^{2}} .
$$

This classical equation has been associated to various Hamiltonians:

(1) In [25], Rouy and Tourin introduce

$$
H_{R / T}^{\text {orth }}(x, p)=I(x) \sqrt{1+|p|^{2}}+p \cdot \mathbf{l}-\gamma .
$$

(2) In [11], Dupuis and Oliensis consider

$$
H_{D / O}^{\text {orth }}(x, p)=I(x) \sqrt{1+|p|^{2}-2 p \cdot \mathbf{l}}+p \cdot \mathbf{l}-1 .
$$

(3) For $\mathbf{L}=(0,0,1)$, Lions et al. [18] deal with the Eikonal equation:

$$
H_{\text {Eiko }}^{\text {orth }}(x, p)=|p|-\sqrt{\frac{1}{I(x)^{2}}-1} .
$$

"Perspective SFS": in [21,28], the camera is modeled as performing a perspective projection. The authors parameterize the surface $\mathbf{S}$ by defining $S(x)=u(x)(x,-\mathbf{f})$, where $\mathbf{f}$ represents the focal length. Setting $v=\ln (u)$, we obtain from the equation (1.1) the following Hamiltonian for unknown $v$

$$
H_{P / F}^{p e r s}(x, p)=I(x) \sqrt{\mathrm{f}^{2}|p|^{2}+(x \cdot p+1)^{2}}-(\mathrm{f} \mathbf{l}+\gamma x) \cdot p-\gamma .
$$




\subsection{Light source at the optical center}

When the light source is located at the optical center, $\mathbf{L}(S(x))=S(x) /|S(x)|$.

This modeling, associated with a perspective projection, corresponds nicely to the situation encountered in some medical protocols like endoscopy, in which the light source is located very close to the camera. It also corresponds approximately to the situation encountered when we use a simple camera equipped with a flash. In [22], we parameterize the surface $\mathbf{S}$ by defining $S(x)=\frac{\mathrm{f} u(x)}{\sqrt{|x|^{2}+\mathrm{f}^{2}}}(x,-\mathrm{f})$. Making the change of variables $v=\ln (u)$, we obtain from the equation (1.1) the following Hamiltonian:

$$
H_{F o c}^{\text {pers }}(x, p)=I(x) \sqrt{\mathfrak{f}^{2}|p|^{2}+(p \cdot x)^{2}+Q(x)^{2}}-Q(x),
$$

where $Q(x)=\sqrt{\mathrm{f}^{2} /\left(|x|^{2}+\mathrm{f}^{2}\right)}$.

\subsection{A generic Hamiltonian}

In [22], we prove that all the previous SFS Hamiltonians are special cases of the following "generic" Hamiltonian:

$$
H_{g}(x, p)=\kappa_{x} \sqrt{\left|A_{x} p+\mathbf{v}_{x}\right|^{2}+K_{x}^{2}}+\mathbf{w}_{x} \cdot p+c_{x},
$$

with $\kappa_{x}, K_{x} \geq 0, c_{x} \in \mathbb{R}, \mathbf{v}_{x}, \mathbf{w}_{x} \in \mathbb{R}^{2}$ and $A_{x}$ is $2 \times 2$ matrix. By using the Legendre transform, we rewrite this "generic" Hamiltonian as a supremum:

$$
H_{g}(x, p)=\sup _{a \in B_{2}(0,1)}\left\{-f_{g}(x, a) \cdot p-l_{g}(x, a)\right\}
$$

where the exact expressions of $f_{g}$ and $l_{g}$ are detailed in [22]. This generic formulation considerably simplifies the analysis of the problem. In particular, this formulation unifies the orthographic and perspective SFS problems. Also, from a practical point of view, a unique algorithm can be used to numerically solve these various problems.

\section{Singular Discontinuous viscosity solutions With Dirichlet Boundary CONDITIONS AND STATE CONSTRAINTS (SDVS)}

The notion of singular viscosity solutions was pioneered by Ishii and Ramaswamy [15] and has been recently upgraded by Camilli and Siconolfi $[6,7]$. In this section, we modify the tools developed in these papers introducing the notion of "singular discontinuous viscosity solution with Dirichlet boundary conditions and state constraints" (SDVS). Since we are mainly interested to the application to SFS equations we will make the assumptions which are natural in this contest, without looking for the maximal generality.

Let $\Omega$ be a bounded open subset of $\mathbb{R}^{N}$ with smooth boundary (say $W^{1, \infty}$ ). In the SFS problem $N=2$, so $\Omega$ is the rectangular domain given by the image. We consider the Hamilton-Jacobi equation

$$
H(x, \nabla u)=0, \quad x \in \Omega,
$$

where the Hamiltonian $H: \bar{\Omega} \times \mathbb{R}^{N} \rightarrow \mathbb{R}$ is continuous and convex (with respect to $\nabla u$ ). Moreover we assume that there exists a subsolution $\psi \in C^{1}(\Omega) \cap W^{1, \infty}(\Omega)$ of (2.1) (i.e.: for any $x \in \Omega, H(x, \nabla \psi(x)) \leq 0$ ) and

$$
\begin{gathered}
\text { for any } \lambda \in(0,1), p \in \mathbb{R}^{N} \text { s.t. } H(x, p) \leq 0 \text { then } \\
H(x, \lambda p+(1-\lambda) \nabla \psi(x))<0 .
\end{gathered}
$$

We say that $\psi \in C^{1}(\Omega)$ is a "classical" strict subsolution of (2.1) at $x$ when $H(x, \nabla \psi(x))<0$. We denote by $\mathcal{S}$ the set of singular points of $H$ respect to $\psi$ :

$$
\mathcal{S}=\{x \in \bar{\Omega} \mid H(x, \nabla \psi(x))=0\},
$$


i.e. the set where $\psi$ fails to be a "classical" strict subsolution of (2.1). $\mathcal{S}$ is closed by the continuity of $\nabla \psi$ and $H$. We assume that

$$
\mathcal{S} \cap \partial \Omega=\emptyset
$$

We recall that if $\mathcal{S}$ is empty then there exists a unique viscosity solution to (2.1) completed with an appropriate boundary condition. If $\mathcal{S}$ is not empty, then in general uniqueness fails.

Remark 2.1. Note that the previous hypotheses on $H$ hold for all the SFS Hamiltonians considered in Section 1 (see [22]) as soon as the image brightness $I$ is continuous and verifies $I(x)>0$ for any $x \in \bar{\Omega}$. In [22], we prove that for all the SFS Hamiltonians presented in Section 1, the set of singular points $\mathcal{S}$ corresponds with the set of "critical points" $\{x \in \bar{\Omega} \mid I(x)=1\}$. Since $I(x)$ is the cosine of the angle $(\mathbf{n}(x), \mathbf{L}(x))$, these points correspond with the ones of maximal brightness.

In this paper, we also assume that the Hamiltonian $H$ satisfies the coercitivity condition

$$
\liminf _{|p| \rightarrow+\infty} H(x, p)=+\infty, \quad \text { for any } x \in \bar{\Omega} .
$$

Remark 2.2. The coercivity hypothesis (2.4) is not systematically verified for all the SFS Hamiltonians. Globally, it does not hold for the pixels of the image with a low brightness, i.e. $I(x)$ close to 0 , when the direction of the distant light source is very different to the one of the camera (see [22]).

Remark 2.3. In the case where $H$ is a coercive Hamilton-Jacobi-Bellman (HJB) Hamiltonian

$$
H(x, p)=\sup _{a \in A}\{-f(x, a) \cdot p-l(x, a)\}
$$

with $f: \bar{\Omega} \times A \rightarrow \mathbb{R}^{N}, l: \bar{\Omega} \times A \rightarrow \mathbb{R}$ and the "cost" $l$ is nonnegative, a subsolution is $\psi \equiv 0$. The corresponding set $\mathcal{S}$ is given by

$$
\{x \in \bar{\Omega} \mid l(x, a)=0 \text { for some } a \in A\} .
$$

Yet, at the opposite of [6], in this paper we do not assume that $l$ is a nonnegative function. As it was shown in [22] (see Sect. 3.5), the Rouy/Tourin Hamiltonian $H_{R / T}^{\text {orth }}$ (where, $l_{R / T}(x, a)=I(x) \sqrt{1+|a|^{2}}-\gamma$ ) and the perspective Hamiltonian $H_{P / F}^{\text {pers }}$, which fit in the class of Hamiltonians given by (2.5) but with a cost of arbitrary sign, admit a regular subsolution. Therefore, in this paper, to each SFS Hamiltonian we systematically associate the corresponding subsolution $\psi$ defined in [22].

We complement (2.1) with a "boundary condition" which represents the part of the data of the surface to reconstruct that we have at our disposal. We consider a function $\varphi: \bar{\Omega} \rightarrow \mathbb{R} \cup\{+\infty\}, \varphi \not \equiv+\infty$, l.s.c., bounded from below and continuous in $\{x \in \bar{\Omega}: \varphi(x)<+\infty\}$. Moreover we assume that the set $K=\{x \in \Omega: \varphi(x)<$ $+\infty\}$ is a (possible empty) subset of $\Omega$ such that

$$
\bar{K} \subset \Omega,
$$

where $\bar{K}$ is the closure of $K$ in $\mathbb{R}^{N}$. The set $K$ represent the available data inside $\Omega$.

With equation (2.1), we associate the "Dirichlet boundary conditions" (DBC)

$$
u(x)=\varphi(x), \quad \forall x \in \bar{\Omega}
$$

(of course, this constraint defined on the whole set $\bar{\Omega}$ must not be considered in pointwise sense).

At points $x \in \partial \Omega$ where $\varphi(x)=+\infty$, we say that we impose a state constraint boundary condition $($ see $[8,26])$. We refer to Remarks 2.15 and 2.16 for some comments about this boundary condition and for the implications in the shape-from-shading problem. 


\subsection{Viscosity subsolutions}

If $u$ be a locally bounded function on a set $E$, we define

$$
\begin{aligned}
& u^{*}(x)=\limsup _{y \rightarrow x} u(y)=\sup \left\{\limsup _{n \rightarrow \infty} u\left(x_{n}\right): x_{n} \in E, x_{n} \rightarrow x,\right\}, \\
& u_{*}(x)=\liminf _{y \rightarrow x} u(y)=\inf \left\{\liminf _{n \rightarrow \infty} u\left(x_{n}\right): x_{n} \in E, x_{n} \rightarrow x\right\}
\end{aligned}
$$

for any $x \in E . u^{*}$ and $u_{*}$ are respectively called the upper semicontinuous envelope and lower semicontinuous envelope of $u$. Recall that, if $u$ is a locally bounded function, then $u^{*}$ is u.s.c and $u_{*}$ is l.s.c. (see [1] for more details).

We now give the definition of viscosity subsolution. The definition of viscosity supersolution, which is modified respect to the standard one to solve the uniqueness issue, is postponed to the next subsection.

Definition 2.4. A locally bounded function $u$, u.s.c in $\Omega$, is said a viscosity subsolution of equation (2.1) if for any $\phi \in C^{1}(\Omega)$, for any $x_{0} \in \Omega$ local maximum of $(u-\phi)$ in $\Omega$, then

$$
H\left(x_{0}, \nabla \phi\left(x_{0}\right)\right) \leq 0
$$

The regularity of $\partial \Omega$ and the hypothesis on $H$ imply that a viscosity subsolution of (2.1) is Lipschitz continuous in $\Omega$ and moreover (see Prop. 4.3 in [6]).

Proposition 2.5. The following three properties are equivalent

- $u$ is a viscosity subsolution of (2.1) in $\Omega$;

- $u$ is a Lipschitz continuous a.e. subsolution of (2.1) in $\Omega$;

- $u$ is Lipschitz continuous and the inequality

$$
H(x, p) \leq 0
$$

holds for any $x \in \Omega, p \in \partial u(x)$ (where $\partial u$ is the (Clarke) generalized gradient [9]).

Remark 2.6. If the Hamiltonian is noncoercive then subsolutions are not necessarily Lipschitz continuous. Moreover, subsolutions can yield discontinuities at the points $x$ where $p \mapsto H(x, p)$ is non coercive. Nevertheless, note that, if $\mathcal{S}$ is empty, only the coercivity on a neighborhood of $\partial \Omega$ is sufficient for ensuring the strong uniqueness, and so the continuity of the discontinuous viscosity solution on $\Omega$ (see for example Thm. 4.5 of [3] and more especially its Cor. 4.1).

Definition 2.7. A locally bounded function $u$, u.s.c. on $\bar{\Omega}$, is said a viscosity subsolution of $(2.1)-(2.7)$ if $u$ is a viscosity subsolution of $(2.1)$ and if:

- For any $x_{0} \in K, \quad u\left(x_{0}\right) \leq \varphi\left(x_{0}\right)$.

- For any $x_{0} \in \partial \Omega$, $\circ u\left(x_{0}\right) \leq \varphi\left(x_{0}\right)$

o or for any $\phi \in C^{1}(\bar{\Omega})$ s.t. $x_{0}$ is a local maximum of $(u-\phi)$ in $\bar{\Omega}, H\left(x_{0}, \nabla \phi\left(x_{0}\right)\right) \leq 0$.

Note that points $x \in \partial \Omega$ where $\varphi(x)=+\infty$, the boundary condition is automatically satisfied.

\subsection{Singular viscosity supersolutions}

Before giving the definition of the singular viscosity supersolution of (2.1)-(2.7), we need to detail various preliminary definitions. 


\subsubsection{The multivalued map}

Let $\mathcal{Z}(x)$ be the multivalued map on $\bar{\Omega}$ defined as:

$$
\mathcal{Z}(x)=\left\{p \in \mathbb{R}^{N}: H(x, p) \leq 0\right\}
$$

For all the SFS Hamiltonians considered in this paper, it is easy to see that:

$$
\mathcal{Z}(x)=\{\nabla \psi(x)\} \quad \text { for any } x \in \mathcal{S} \text {. }
$$

Therefore, in the sequel, we assume that hypothesis (2.9) holds.

Remark 2.8. Under the hypothesis (2.9), the continuity of $H$ provides a new characterization of $\mathcal{S}$ :

$$
x \in \mathcal{S} \Longleftrightarrow \mathcal{Z}(x)=\{\nabla \psi(x)\}
$$

Assumption (2.9) and Proposition 2.5 imply that for a subsolution $u, \partial u(x)=\{\nabla \psi(x)\}$ for $x \in \mathcal{S}$. Therefore a subsolution $u$ is strictly differentiable (see [9]) on the singular set.

By (2.4) and (2.2), the set-valued map $\mathcal{Z}(x)$ is continuous in $\bar{\Omega}$ respect to the Hausdorff metric. Moreover, for any $x \in \bar{\Omega}$, the set $\mathcal{Z}(x)$ is compact, convex, strictly star-shaped respect to $\nabla \psi(x)$ and

$$
\partial \mathcal{Z}(x)=\left\{p \in \mathbb{R}^{N} \mid H(x, p)=0\right\} .
$$

\subsubsection{A new Hamiltonian with the same multivalued map}

Now, let us introduce the gauge function $\rho(x, p)$ of $\mathcal{Z}(x)$. We set for any $x \in \bar{\Omega}, p \in \mathbb{R}^{N}$,

$$
\rho(x, p)=\inf \left\{\lambda>0: \lambda^{-1} p+\left(1-\lambda^{-1}\right) \nabla \psi(x) \in \mathcal{Z}(x)\right\}
$$

As in $[2,3]$, (see also Prop. 5.1 of [7]), we can prove that the function $\rho$ is l.s.c in $\bar{\Omega} \times \mathbb{R}^{N}$ (continuous in $\left.(\bar{\Omega} \backslash \mathcal{S}) \times \mathbb{R}^{N}\right)$ and verifies the homogeneity condition:

$$
\rho(x, \mu p+(1-\mu) \nabla \psi(x))=\mu \rho(x, p)
$$

for any $\mu>0$ and for any $(x, p) \in \bar{\Omega} \times \mathbb{R}^{N}$.

Note that $H(x, p) \leq 0$ if and only if $\rho(x, p) \leq 1$, hence

$$
\mathcal{Z}(x)=\left\{p \in \mathbb{R}^{N}: \rho(x, p) \leq 1\right\} .
$$

If $x \in \mathcal{S}$, we have $\rho(x, \nabla \psi(x))=0$ and $\rho(x, p)$ is infinite for $p \neq \nabla \psi(x)$.

Remark 2.9. The multiplicity of the classical viscosity solutions is related to the fact that the subsolution $\psi$ fails to be strict at the points $x \in \mathcal{S}$. To get round this difficulty the idea consists in transforming the equation $H(x, \nabla u)=0$ in the new equation $\rho(x, \nabla u)=1$. This equation is equivalent to (2.1) on $\Omega \backslash \mathcal{S}$ and for any $x \in \mathcal{S}, \rho(x, \nabla \psi(x))=0<1$, i.e. $\psi$ is a strict subsolution of the new equation on $\mathcal{S}$.

\subsubsection{Adaptation of the topology}

Let us set, for any $x \in \bar{\Omega}$,

$$
r(x)=\sup \{r>0 \mid B(\nabla \psi(x), r) \subset \mathcal{Z}(x)\} .
$$

In [7], Lemma 3.1 it is proved that $r(x)$ is continuous in $\bar{\Omega}, r(x)$ is nonnegative and $r(x)=0$ if and only if $x \in \mathcal{S}$. Note that by definition of $r($.$) and (2.9)$ we have

- $x \in \mathcal{S} \Longleftrightarrow \mathcal{Z}(x)=\{\nabla \psi(x)\} \Longleftrightarrow r(x)=0$;

- $x \notin \mathcal{S} \Longleftrightarrow \exists r>0$ s.t. $B(\nabla \psi(x), r) \subset \mathcal{Z}(x) \Longleftrightarrow r(x)>0$. 
We proceed defining a semidistance on $\bar{\Omega}$. We set for any $x, y \in \bar{\Omega}$,

$$
\begin{gathered}
S(x, y)=\inf \left\{\int_{0}^{1} r(\xi(t))|\dot{\xi}(t)| \mathrm{d} t: \xi(t) \in W^{1, \infty}([0,1], \bar{\Omega})\right. \\
\text { s.t. } \xi(0)=x \text { and } \xi(1)=y\} .
\end{gathered}
$$

It is easy to verify that $S$ satisfies:

$$
\begin{gathered}
S(x, y) \leq S(x, z)+S(z, y) \quad x, y, z \in \bar{\Omega} \\
S(x, y)=S(y, x) \quad x, y \in \bar{\Omega} \\
S(x, x)=0 \quad x \in \bar{\Omega}
\end{gathered}
$$

and

$$
0 \leq S(x, y) \leq\|r\|_{\infty} d_{E}(x, y) \quad x, y \in \bar{\Omega}
$$

where $d_{E}(x, y)$ is the Euclidean geodesic distance in $\Omega$ (i.e. the distance defined as in (2.13) with $\left.r(x) \equiv 1\right)$.

So $S$ is a semidistance on $\bar{\Omega}$, but in general not a distance. The set of points which have null $S$-distance from $x_{0} \in \mathcal{S}$ is in general a subset of $\mathcal{S}$ containing elements different from $x_{0}$. The family of balls:

$$
B_{S}\left(x_{0}, R\right)=\left\{x \in \Omega \mid S\left(x_{0}, x\right) \leq R\right\}
$$

induces a topology $\tau_{S}$ in $\Omega$. Note that in a neighborhood of a point $x \in \Omega \backslash \mathcal{S}$ the topology $\tau_{S}$ is equivalent to the Euclidean topology. At a point $x \in \mathcal{S}$, it is a weaker topology. We denote by $B_{S}\left(x_{0}\right)$ the subset

$$
B_{S}\left(x_{0}\right)=\left\{x \in \bar{\Omega} \mid S\left(x_{0}, x\right)=0\right\} .
$$

\subsubsection{Definition of singular viscosity supersolutions and solutions}

We need two prelimiry definitions

Definition 2.10. A function $v$ is said to be a strict subsolution of (2.1) in an open subset $A$ of $\Omega$ if $v$ is a viscosity subsolution of

for some $\theta \in(0,1)$.

$$
\rho(x, \nabla v) \leq \theta \quad x \in A
$$

Definition 2.11. For a l.s.c. function $v$, a Lipschitz continuous function $\phi$ is called $S$-subtangent to $v$ at $x_{0} \in \Omega$ if $x_{0}$ is a minimizer of $v-\phi$ in a $\tau_{S}$-neighborhood of $x_{0}$ (or equivalently, in a neighborhood $A$ of $B_{S}\left(x_{0}\right)$ ). The $S$-subtangent is called strict if the inequality

$$
(v-\phi)(x)>(v-\phi)\left(x_{0}\right)
$$

holds for $x \in A \backslash B_{S}\left(x_{0}\right)$.

Observe that an $S$-subtangent is also a subtangent in the standard viscosity solution sense, but the converse is not true. We now give the definition of (singular) viscosity supersolution for (2.1)

Definition 2.12. A 1.s.c. function $v: \Omega \rightarrow \mathbb{R}$ is said singular viscosity supersolution of (2.1) at $x_{0} \in \Omega$, if it does not admit a $S$-subtangent at $x_{0}$ which is a strict subsolution of (2.1) in a neighborhood of $B_{S}\left(x_{0}\right)$.

It is worth noting that if $x_{0} \notin \mathcal{S}$, the previous definition is equivalent to the standard notion of viscosity supersolution of equation (2.1) (recall that a locally bounded function $v$, l.s.c in $\Omega$, is said a viscosity supersolution if for any $\phi \in C^{1}(\Omega)$ and for any $x_{0} \in \Omega$ local minimum of $(u-\phi)$, then $\left.H\left(x_{0}, \nabla \phi\left(x_{0}\right)\right) \geq 0\right)$. 
In other respects, if $u$ is a viscosity solution of (2.1), it is possible to prove (see for example [17]) that, on each regular connected component $\mathcal{S}_{i}$ of $\mathcal{S}$, there exists $c_{i}$ such that

$$
u(x)=\psi(x)+c_{i} \quad \text { for any } x \in \mathcal{S}_{i},
$$

(in [6], a counterexample based on the classical Whitney's function shows that if $\mathcal{S}_{i}$ is not regular the previous property can be false). So, if $x_{0} \in \operatorname{Int}\left(\mathcal{S}_{i}\right)$, then $\psi$ is always a subtangent to $u$ at $x_{0}$ and a strict subsolution of the new equation $\rho(x, \nabla u(x))=1$. If we want to preserve on the singular set the property that a strict subsolution cannot be subtangent to a solution, we need to use the weaker topology $\tau_{S}$ in such a way that all the neighborhoods of $x_{0} \in \operatorname{Int}\left(\mathcal{S}_{i}\right)$ contain the whole subset $\mathcal{S}_{i}$. Thus $\psi$ would be not systematically subtangent to any solution.

Definition 2.13. A locally bounded function $v: \bar{\Omega} \rightarrow \mathbb{R}$, l.s.c. on $\bar{\Omega}$, is said singular viscosity supersolution of (2.1)-(2.7) if:

- For any $x_{0} \in \Omega \backslash K, v$ is a singular viscosity supersolution of (2.1) at $x_{0}$.

- For any $x_{0} \in \partial \Omega \cup K$,

$-v$ is a singular viscosity supersolution of $(2.1)$ at $x_{0}$

- or there exists $x \in B_{S}\left(x_{0}\right)$ such that $v\left(x_{0}\right) \geq \varphi(x)+\psi\left(x_{0}\right)-\psi(x)$.

Let us emphasize that, if the set of singular points $\mathcal{S}$ and $K$ are empty, then the singular supersolutions of (2.1)-(2.7) coincide with the standard discontinuous viscosity supersolutions of (2.1)-(2.7). Let us also remind that if $x_{0} \in \mathcal{S}$, then $B_{S}\left(x_{0}\right)$ can be larger than $\left\{x_{0}\right\}$. As the definition of supersolution, also the boundary condition on $K \cap \mathcal{S}$ is adapted to the weak topology induced by the semidistance $S$.

Now, we can give the definition of the singular viscosity solution of (2.1)-(2.7).

Definition 2.14. A locally bounded function $u: \bar{\Omega} \rightarrow \mathbb{R}$ is said singular viscosity solution of (2.1)-(2.7) if $u^{*}$ is a subsolution of (2.1)-(2.7) and if $u_{*}$ is a singular supersolution of (2.1)-(2.7).

We will call "singular discontinuous viscosity solutions with Dirichlet boundary conditions and state constraints" (SDVS), the singular solution of (2.1)-(2.7).

Remark 2.15. A contribution of the notion of SDVS lies in the possibility to impose the heights of the solution at the interior points and on the boundary of the image where we know the data otherwise to send them to infinity. Therefore, in contrast with the classical Dirichlet and Neumann boundary conditions, which require the complete knowledge of the solution on the boundary, we need to know the solution only on a closed subset of $K \cup \partial \Omega$. We then characterize the maximal solution associated with the known data. Let us remind that with discontinuous viscosity solutions it is ineffective to impose the height of the solution the singular points (such a constraint cannot allow to characterize a solution), see [22]. This is possible with continuous viscosity solutions but compatibility conditions are required, see [25].

Remark 2.16. Note that, in [18], Lions et al. have already used the notion of states constraints, but they used it only to deal with apparent contours and in the eikonal setup. More precisely, they use it at the points $x \in \partial \Omega$ such that $I(x)=0$ and " $\frac{\partial u}{\partial n}=-\infty$ ".

We recall also an interesting interpretation of boundary condition when the solution is differentiable and the Hamiltonian is increasing in $p$ (see [8]). In this case we obtain

$$
\frac{\partial u}{\partial n} \geq 0
$$

at the points of the boundary where the state-constraint is verified ( $n$ is the external normal). This means that $u$ increases when comes up to the boundary. 


\section{Existence And uniqueness of SDVS}

In this section, we prove the existence and uniqueness of the SVDS for the shape-from-shading problems.

\subsection{Existence and representation of SDVS}

We prove the existence of SDVS by giving an explicit representation formula of it. Let $\delta: \bar{\Omega} \times \mathbb{R}^{N} \rightarrow \mathbb{R}$ be the support function of the set $\tilde{\mathcal{Z}}(x)=\mathcal{Z}(x) \backslash \nabla \psi(x)$, i.e.:

$$
\delta(x, p)=\max \{p q: q \in \tilde{\mathcal{Z}}(x)\} .
$$

The function $\delta(x, p)$ is continuous in $\bar{\Omega} \times \mathbb{R}^{N}$, convex and positively homogeneous in $p$. Moreover there exists $R$ such that

$$
0 \leq \delta(x, p) \leq R|p| \quad x \in \bar{\Omega}, p \in \mathbb{R}^{N}
$$

(see [7]). For $A \subset \bar{\Omega}$, we denote for any $x, y \in \bar{A}$,

$$
\begin{aligned}
& L_{A}(x, y)=\inf \left\{\int_{0}^{1} \delta(\xi(t),-\dot{\xi}(t)) \mathrm{d} t \mid \xi(t) \in W^{1, \infty}([0,1], \bar{A})\right. \\
& \text { s.t. } \quad \xi(0)=x \text { and } \xi(1)=y\}
\end{aligned}
$$

and we set $L(x, y):=L_{\Omega}(x, y)$. From (3.2) it follows that $0 \leq L(x, y) \leq R d_{E}(x, y)$ and therefore $y \mapsto L(x, y)$ is Lipschitz continuous in $\bar{\Omega}$ for any fixed $x \in \bar{\Omega}$ (with a Lipschitz constant which does not depend on $x$ ). We recall that (see [6])

Proposition 3.1. $u$ is a subsolution of (2.1) in $\Omega$ if and only if

$$
u(x) \leq \psi(x)+u(y)-\psi(y)+L(x, y) \quad \text { for any } x, y \in \Omega .
$$

Remark 3.2. If $x \in \mathcal{S}$, then $\tilde{\mathcal{Z}}(x)=\{0\}$ and therefore $\delta(x, p)=0$ for any $p \in \mathbb{R}^{N}$. Also, the inverse statement holds. Hence

So for any $x, y \in \bar{\Omega}$,

$$
\delta(x, p)=0 \quad \text { for any } p \in \mathbb{R}^{N} \Longleftrightarrow x \in \mathcal{S} \Longleftrightarrow r(x)=0 .
$$

In other words,

$$
L(x, y)=0 \Longleftrightarrow S(x, y)=0 .
$$

$$
B_{S}\left(x_{0}\right)=\left\{x \in \Omega: L\left(x_{0}, x\right)=0\right\} \quad \text { for any } x_{0} \in \bar{\Omega} .
$$

Remark 3.3. When the Hamiltonian is not coercive, it may exist $x \in \bar{\Omega}$ such that $\mathcal{Z}(x)$ is unbounded. Hence for some $p \neq \nabla \psi(x)$, we may have $\rho(x, p)=0$ and $\delta(x, p)=+\infty$. So, for some $(x, y) \in \bar{\Omega} \times \bar{\Omega}$, we can have $L(x, y)=+\infty$. Note that, since $L$ is in general nonsymmetric, it can result $L(y, x)<+\infty$.

Let us consider the function

$$
V(x)=\psi(x)+\min \{L(x, y)+\varphi(y)-\psi(y) \mid y \in \partial \Omega \cup K\} .
$$

Theorem 3.4. The function $V$ is a singular solution of (2.1)-(2.7).

To see that $V$ is a viscosity subsolution of (2.1), we observe that the constraint $V(x) \leq \varphi(x)$ for $x \in K$ is satisfied. Moreover since for any $x_{1}, x_{2} \in \Omega$

$$
V\left(x_{1}\right)-V\left(x_{2}\right) \leq \psi\left(x_{1}\right)-\psi\left(x_{2}\right)+L\left(x_{1}, y_{1}\right)-L\left(x_{2}, y_{1}\right) \leq \psi\left(x_{1}\right)-\psi\left(x_{2}\right)+L\left(x_{1}, x_{2}\right)
$$

from Proposition 3.3 it follows that $V$ is a subsolution in $\Omega$. Finally, to prove the subsolution condition at the boundary we can argue as in [8] (recall assumption (2.3)).

To show that $V$ is a singular supersolution of $(2.1)-(2.7)$, we need some preliminary results. 
Proposition 3.5. Set

$$
\Gamma_{V}=\left\{x \in K \mid V(x) \geq \varphi(y)+\psi(x)-\psi(y) \text { for some } y \in B_{S}(x)\right\}
$$

If $x_{0} \in \Omega \backslash \Gamma_{V}$, then $B_{S}\left(x_{0}\right) \cap \Gamma_{V}=\emptyset$.

Proof. The statement is obvious if $x_{0} \notin \mathcal{S}$, since in this case $B_{S}\left(x_{0}\right)=\left\{x_{0}\right\}$. If $x_{0} \in \mathcal{S} \cap K$, assume by contradiction that there exists $x_{1} \in B_{S}\left(x_{0}\right) \cap \Gamma_{V}$. Hence there exists $y \in B_{S}\left(x_{1}\right)$ such that $V\left(x_{1}\right) \geq \varphi(y)+$ $\psi\left(x_{1}\right)-\psi(y)$. Since $S\left(x_{0}, y\right) \leq S\left(x_{0}, x_{1}\right)+S\left(x_{1}, y\right)=0, y \in B_{S}\left(x_{0}\right)$. By (3.4), (3.3) and $x_{0} \notin \Gamma_{V}$ we have

$$
V\left(x_{1}\right) \leq V\left(x_{0}\right)+\psi\left(x_{1}\right)-\psi\left(x_{0}\right)<\varphi(y)+\psi\left(x_{0}\right)-\psi(y)+\psi\left(x_{1}\right)-\psi\left(x_{0}\right)
$$

and so

hence a contradiction.

$$
V\left(x_{1}\right)<\varphi(y)+\psi\left(x_{1}\right)-\psi(y)
$$

Remark 3.6. $\Gamma_{V}$ is the subset of $K$ where $V$ takes the boundary datum $\varphi$ (in the sense of the topology $\tau_{S}$ on $\mathcal{S} \cap K)$. In $\Omega \backslash \Gamma_{V}, V$ satisfies the supersolution condition.

Proposition 3.7 (Dynamic programming principle). For all $x \in \Omega \backslash \Gamma_{V}$ and all $\tau_{S}$-neighborhood $A$ of $x$ s.t. $A \cap\left(\Gamma_{V} \cup \partial \Omega\right)=\emptyset$,

$$
V(x)=\psi(x)+\min _{y \in \partial A}\{L(x, y)+V(y)-\psi(y)\} .
$$

Proof. Classic; see for example [1].

Proposition 3.8. Let $u$ be a l.s.c. function, $\phi_{0}$ a S-subtangent to $u$ at a point $x_{0}$ and a strict subsolution of (2.1) in a $\tau_{S}$-neighborhood of $x_{0}$. Then there exists a function $\phi$ which is strict $S$-subtangent to $u$ at $x_{0}$ and a strict subsolution of (2.1) in a $\tau_{S}$-neighborhood of $x_{0}$ and such that for any $x \in \Omega, q \in \partial \phi(x)$, one can select $p \in \partial \phi_{0}(x)$ verifying

$$
\rho(x, q) \leq \rho(x, p)+L\left(x_{0}, x\right) .
$$

Proof. See the proofs of Proposition 6.1 of [7] and of Proposition 5.1 of [6].

Proof of Theorem 3.4. We first consider the case $x_{0} \in \Omega \backslash \Gamma_{V}$ and we argue by contradiction. Let us assume that there exists a function $\phi_{0}$, a neighborhood $A$ of $B_{S}\left(x_{0}\right)$ and $\left.\theta \in\right] 0,1\left[\right.$ s.t. $\phi_{0}$ is a $S$-subtangent to $V$ at $x_{0}$ with $\phi_{0}\left(x_{0}\right)=V\left(x_{0}\right)$ and

$$
\rho\left(x, \nabla \phi_{0}\right) \leq \theta, \quad x \in A
$$

in the viscosity sense.

Let $\phi$ be a strict $S$-subtangent to $V$ at $x_{0}$ verifying the statement of Proposition 3.8. By continuity of the function $x \mapsto L\left(x_{0}, x\right)$ and (3.4), we can select a neighborhood $A^{\prime}$ of $B_{S}\left(x_{0}\right)$ and with $\overline{A^{\prime}} \subset A$ satisfying

$$
\begin{array}{r}
\sup _{x \in A^{\prime}} L\left(x_{0}, x\right)<1-\theta, \\
\phi<V \text { on } \partial A^{\prime} \\
A^{\prime} \cap\left(\Gamma_{V} \cup \partial \Omega\right)=\emptyset .
\end{array}
$$

Since $x_{0} \in \Omega \backslash \Gamma_{V}$, we can assume the dynamic programming principle (3.6) holds on $A^{\prime}$, so there exists $y_{0} \in \partial A^{\prime}$ such that

By

$$
V\left(x_{0}\right)=\psi\left(x_{0}\right)+L_{A^{\prime}}\left(x_{0}, y_{0}\right)+V\left(y_{0}\right)-\psi\left(y_{0}\right)
$$

we get

$$
V\left(x_{0}\right)=\phi\left(x_{0}\right) \quad \text { and } \quad V\left(y_{0}\right)>\phi\left(y_{0}\right)
$$

$$
L_{A^{\prime}}\left(x_{0}, y_{0}\right)+\left[\phi\left(y_{0}\right)-\psi\left(y_{0}\right)\right]-\left[\phi\left(x_{0}\right)-\psi\left(x_{0}\right)\right]<0 .
$$


So we can select a path $\xi \in W^{1, \infty}\left([0,1], \overline{A^{\prime}}\right)$ joining $x_{0}$ to $y_{0}$ satisfying

$$
\int_{0}^{1}\left[\delta(\xi(t),-\dot{\xi}(t))+\frac{\mathrm{d}}{\mathrm{d} t}(\phi(\xi(t))-\psi(\xi(t)))\right] \mathrm{d} t<0 .
$$

Hence there exists $t_{0} \in[0,1]$ such that the functions $\phi(\xi(t))$ and $\xi(t)$ are differentiable at $t_{0}$ and

$$
\delta\left(\xi\left(t_{0}\right),-\dot{\xi}\left(t_{0}\right)\right)-\dot{\xi}\left(t_{0}\right) \nabla \psi\left(\xi\left(t_{0}\right)\right)+\frac{\mathrm{d}}{\mathrm{d} t} \phi\left(\xi\left(t_{0}\right)\right)<0 .
$$

Using the chain rule for the generalized gradient (see [9]), we derive from (3.10)

$$
\delta\left(\xi\left(t_{0}\right),-\dot{\xi}\left(t_{0}\right)\right)<-\dot{\xi}\left(t_{0}\right)\left(q_{0}-\nabla \psi\left(\xi\left(t_{0}\right)\right)\right)
$$

for some $q_{0} \in \partial \phi\left(\xi\left(t_{0}\right)\right)$. Hence

$$
\rho\left(\xi\left(t_{0}\right), q_{0}\right)>1
$$

Therefore by (3.7) and (3.9) the inequality

$$
\rho\left(\xi\left(t_{0}\right), p_{0}\right)>\theta
$$

holds for a suitable $p_{0} \in \partial \phi_{0}\left(\xi\left(t_{0}\right)\right)$. This contradicts (3.8) and Proposition 2.5.

If $x_{0} \in \Gamma_{V}$, by definition of $\Gamma_{V}$, we have $V\left(x_{0}\right) \geq \varphi(x)+\psi\left(x_{0}\right)-\psi(x)$ for some $x \in B_{S}\left(x_{0}\right)$.

Finally if $x_{0} \in \partial \Omega$, assumption (2.3) implies that there exists a neighborhood $A$ of $\partial \Omega$ in $\bar{\Omega}$ such that $A \cap \mathcal{S}=\emptyset$. If $V\left(x_{0}\right)<\varphi\left(x_{0}\right)$, assuming that the condition of viscosity supersolution does not hold, we can obtain a contradiction by adapting the proof of Theorem V.4.13 of [1].

\subsection{Uniqueness results}

In this section we prove the uniqueness of the SDVS. This result applies for all the shape-from-shading equations described in Section 1. We start this section with a maximum principle:

Theorem 3.9. Let $u, v: \Omega \rightarrow \mathbb{R}$ be respectively an u.s.c. subsolution of (2.1) and a l.s.c. singular supersolution of (2.1)-(2.7). Let us denote

$$
\Gamma_{v}=\left\{x \in K \mid v(x) \geq \varphi(y)+\psi(x)-\psi(y) \text { for some } y \in B_{S}(x)\right\} .
$$

Then

$$
\min _{\bar{\Omega}}\{v-u\}=\min _{\partial \Omega \cup \Gamma_{v}}\{v-u\} .
$$

Proof. Given $\theta \in] 0,1\left[\right.$, the function $u_{\theta}=\theta u+(1-\theta) \psi(x)$ is a strict subsolution of (2.1) in $\Omega$ by the homogeneity of $\rho$ (see (2.12)). Let us assume that $x_{0} \in \Omega \backslash \Gamma_{v}$ is a minimizer of $\left(v-u_{\theta}\right)$ in $\bar{\Omega}$. Therefore $u_{\theta}$ is a $S$-subtangent of $v$ at $x_{0}$ which is also a strict subsolution of (2.1) in $\Omega$. This contradicts that $v$ is a singular supersolution at $x_{0}$. So the minimizers of $\left(v-u_{\theta}\right)$ are in $\partial \Omega \cup \Gamma_{v}$. The assertion is obtained by letting $\theta$ to go to 1 .

In the sequel, we assume that $\partial \Omega \in W^{2, \infty}$ and there exists a neighborhood $A$ of $\partial \Omega$ and $\lambda>0$ such that:

$$
|H(x, p)-H(x, q)| \leq \lambda|p-q| \quad \text { for any } x \in A, \quad \text { for any } p, q \in \mathbb{R}^{N} .
$$

In other words, we impose that $H$ is Lipschitz continuous in $p$ (with a Lipschitz constant which does not depend on $x \in A$ ) on a neighbourhood of $\partial \Omega$. Note that the SFS Hamiltonians $H_{\text {pers }}^{*}$ and $H_{\text {orth }}^{*}$ verify the hypothesis (3.11) (see [22]).

Using the maximum principle, we deduce the following strong uniqueness result for the SDVS with $\varphi \equiv+\infty$ on $\partial \Omega$. 
Theorem 3.10. Let $u, v: \Omega \rightarrow \mathbb{R}$ be respectively an u.s.c. subsolution of (2.1)-(2.7), and a l.s.c. singular supersolution of (2.1)-(2.7), with $\varphi$ verifying $\varphi(x)=+\infty$ for any $x \in \partial \Omega$. If $H$ verifies (3.11) then

$$
u(x) \leq v(x) \text { for any } x \in \Omega .
$$

Proof. We consider $M=\max _{\bar{\Omega}}\{u(x)-v(x)\}$. We argue by contradiction and assume that $M>0$. By the maximum principle (Thm. 3.9), we have:

$$
M=\max _{x \in \bar{\Omega}}\{u(x)-v(x)\}=\max _{x \in \partial \Omega \cup \Gamma_{v}}\{u(x)-v(x)\} .
$$

Let $x \in \Gamma_{v}$ and $y \in B_{S}(x)$ be such that $v(x) \geq \varphi(y)+\psi(x)-\psi(y)$. By (3.3) and because $\Gamma_{v} \subset K$

$$
v(x) \geq \varphi(y)+\psi(x)-\psi(y) \geq u(y)+\psi(x)-\psi(y) \geq u(x) .
$$

So, for any $x \in \Gamma_{v}, u(x)-v(x) \leq 0$ and therefore

$$
M=\max _{x \in \bar{\Omega}}\{u(x)-v(x)\}=\max _{x \in \partial \Omega}\{u(x)-v(x)\} .
$$

In other words, $M$ is reached at a point $x_{0} \in \partial \Omega$. Henceforth, we can work on a neighborhood $A$ of the boundary $\partial \Omega$ where hypothesis (3.11) holds. By (2.3) and (2.6) we can assume that $A \cap(\mathcal{S} \cup K)=\emptyset$. So in this neighborhood $A$, the notion of singular viscosity solution coincides with the classical notion of discontinuous viscosity solutions. Therefore, we can obtain a contradiction exactly as in the proof of Theorem 4.6 of [3] (let us recall that we have assumed that $H$ is coercive in $p$ uniformly with respect to $x$ ).

More generally, we have

Theorem 3.11. Let $u, v: \Omega \rightarrow \mathbb{R}$ be respectively an u.s.c. subsolution of (2.1)-(2.7), and a l.s.c. singular supersolution of (2.1)-(2.7). Then

$$
u(x) \leq v(x) \quad \text { for any } x \in \Omega
$$

Proof. The statement can be proved combining the proofs of the previous theorem and of Theorem 4.5 (and of its Cor. 4.1) of Barles' book [3].

Let us note that clearly the strong uniqueness involves the uniqueness on $\Omega$ of the singular viscosity solution of (2.1)-(2.7): i.e., if $u_{1}$ and $u_{2}$ are two singular viscosity solutions of $(2.1)-(2.7)$, then for any $x \in \Omega, u_{1}(x)=$ $u_{2}(x)$ (recall that instead uniqueness in $\bar{\Omega}$ does not hold [3]). Moreover, it proves that this solution is continuous on $\Omega\left(u=u^{*}=u_{*}\right)$, therefore it is Lipschitz continuous on $\Omega$ (because subsolutions are Lipschitz continuous).

\section{Stability of the Singular SOlution}

Since discontinuous viscosity solutions of (2.1)-(2.7) are in general not unique, standard stability results in viscosity solution theory do not apply. In contrast, in this section we show that, as consequence of the strong comparison result of Section 3.2, the notion of SDVS enjoys some significative stability properties. This fact has, as we show in the next section, important and appreciable consequences for the shape-from-shading problem.

We consider for $n \in \mathbb{N}$ the equations:

$$
H_{n}(x, \nabla u)=0, \quad \text { for any } x \in \Omega
$$

with continuous, convex and coercive Hamiltonians $H_{n}$ satisfying (2.2)-(2.4). We set for any $x \in \bar{\Omega}$

$$
\mathcal{Z}_{n}(x)=\left\{p \in \mathbb{R}^{N} \mid H_{n}(x, p) \leq 0\right\}
$$


We require the following conditions:

$$
\begin{gathered}
\text { there exists } M>0 \text { s.t. } \mathcal{Z}_{n}(x) \subset B(0, M) \text { for any } x \in \bar{\Omega}, n \in \mathbb{N}, \\
\theta_{n} \mathcal{Z}(x)+\left(1-\theta_{n}\right) \nabla \psi(x) \subset \mathcal{Z}_{n}(x) \text { for any } x \in \bar{\Omega}, n \in \mathbb{N}, \\
H(x, p) \leq \liminf _{n \rightarrow+\infty} H_{n}(x, p) \quad \text { for any }(x, p) \in \bar{\Omega} \times B(0, M),
\end{gathered}
$$

where $\theta_{n}$ is a sequence converging to 1 and $\liminf _{*} H_{n}(x, p)=\inf \left\{\liminf H_{n} H_{n}\left(x_{n}, p_{n}\right):\left(x_{n}, p_{n}\right) \rightarrow(x, p)\right\}$.

Remark 4.1. Assumption (4.2) implies that the SDVSs $u_{n}$ of (4.1)-(2.7) verify $\left\|\nabla u_{n}\right\|_{\infty} \leq M$, for any $n$. So the functions $u_{n}$ are uniformly Lipschitz continuous and also uniformly bounded on $\bar{\Omega}$.

Remark 4.2. By (2.9) and Remark 2.8, (4.3) involves

$$
\begin{aligned}
& \mathcal{S}_{n}:=\left\{x \in \bar{\Omega} \mid H_{n}(x, \nabla \psi(x))=0\right\} \subset \mathcal{S} \\
& \theta_{n} S(x, y) \leq S_{n}(x, y), \quad \text { for any } x, y \in \bar{\Omega},
\end{aligned}
$$

where $S_{n}$ is the distance defined as in (2.13) with

$$
r_{n}(x)=\sup \left\{r>0 \mid B(\nabla \psi(x), r) \subset \mathcal{Z}_{n}(x)\right\}
$$

in place of $r($.$) . In particular, the topology \tau_{S_{n}}$ is stronger than the topology $\tau_{S}$.

We have the following stability result:

Theorem 4.3. Let $u_{n}: \bar{\Omega} \rightarrow \mathbb{R}$ be a sequence of solution of (4.1)-(2.7). Assume that (4.2), (4.3) and (4.4) are satisfied. If $u$ is the SDVS of (2.1)-(2.7), then

$$
u(x)=\lim _{n \rightarrow \infty} u_{n}(x)
$$

uniformly in $\bar{\Omega}$.

Proof. We use the semi-relaxed limit technique introduced by Barles and Perthame (see $[1,4]$ ) . We set

$$
\begin{aligned}
\liminf _{*} u_{n}(x) & =\inf \left\{\liminf _{n \rightarrow \infty} u_{n}\left(x_{n}\right): x_{n} \rightarrow x, x_{n} \in \bar{\Omega}\right\}, \\
\limsup { }^{*} u_{n}(x) & =\sup \left\{\limsup _{n \rightarrow \infty} u_{n}\left(x_{n}\right): x_{n} \rightarrow x, x_{n} \in \bar{\Omega}\right\} .
\end{aligned}
$$

By (4.2) the sequence $u_{n}$ is uniformly bounded and uniformly Lipschitz continuous in $\bar{\Omega}$. Hence, all the subsequences of $\left(u_{n}\right)_{n \in \mathbb{N}}$ converging toward limsup* $u_{n}$ and $\liminf _{*} u_{n}$ converge uniformly and limsup* $u_{n}$ and $\liminf _{*} u_{n}$ are bounded and Lipschitz continuous on $\bar{\Omega}$. By (4.4) (see for example [1,2]), it follows that limsup* $u_{n}$ is a viscosity subsolution of (2.1)-(2.7), i.e. it is a subsolution in $\Omega$, satisfies the constraint given by $\varphi$ on $K$ and satisfies the boundary condition on $\partial \Omega$ in viscosity sense.

If $\liminf _{*} u_{n}$ is a singular supersolution of (2.1)-(2.7), then by Theorem 3.11, we get

$$
\limsup ^{*} u_{n} \leq \liminf _{*} u_{n} .
$$

Since the reverse inequality is true by definition we get that $\lim \sup ^{*} u_{n}=\liminf _{*} u_{n}$ and therefore the uniform convergence of the sequence $u_{n}$ toward the SDVS of (2.1)-(2.7). So, to conclude, it is sufficient to prove that all the limits $u$ of subsequences of $u_{n}$ uniformly convergent are singular supersolutions of (2.1)-(2.7). We distinguish two cases:

- 1st case: Assume either that $x_{0} \in K$

$$
u\left(x_{0}\right)<\varphi(y)+\psi\left(x_{0}\right)-\psi(y) \text { for any } y \in B_{S}\left(x_{0}\right)
$$


or that $x_{0} \in \mathcal{S} \backslash K$, otherwise the conclusion is obvious. Note that, by continuity of $u, \varphi$ and $\psi$, inequality (4.5) holds on a neighborhood of $B_{S}\left(x_{0}\right)$. By (4.3), we have

$$
\rho_{n}(x, p) \leq \theta_{n} \rho(x, p)
$$

for any $n \in \mathbb{N}, x \in \Omega$ and $p \in \mathbb{R}^{N}$. Assume for purpose of contradiction that there is a strict $S$-subtangent $\phi$ to $u$ at $x_{0}$ which is also a strict viscosity subsolution of (2.1) in a neighborhood $A$ of $B_{S}\left(x_{0}\right)$, i.e.

$$
\begin{gathered}
(u-\phi)(y)>(u-\phi)\left(x_{0}\right), \text { for any } y \in A \backslash B_{S}\left(x_{0}\right), \\
\rho(y, \nabla \phi(y)) \leq \eta
\end{gathered}
$$

in $A$ in the viscosity sense, for some $\eta \in(0,1)$.

A standard argument in viscosity solution theory gives the existence of a sequence $x_{n}$ of minimizer of $u_{n}-\phi$ verifying $S\left(x_{0}, x_{n}\right) \rightarrow 0$ (see [3], Lem. 4.2). By the uniform convergence of $\left(u_{n}\right)_{n \in \mathbb{N}}$, we have that $u_{n}\left(x_{n}\right)<$ $\varphi(y)+\psi\left(x_{n}\right)-\psi(y)$ for any $y \in B_{S_{n}}\left(x_{n}\right) \subset B_{S}\left(x_{n}\right) \subset A$ for $n$ sufficiently large. Since $A$ is a neighborhood of $B_{S_{n}}\left(x_{n}\right), \phi$ is $S_{n}$-subtangent to $u_{n}$ at $x_{n}$. (4.6) and (4.7) involve:

$$
\rho_{n}(y, \nabla \phi(y)) \leq \theta_{n} \eta, \quad \text { for any } y \in A,
$$

in viscosity sense. Hence $\phi$ is a strict subsolution of (4.1) for $n$ large enough. This contradicts $u_{n}$ being a singular supersolution of (4.1) at $x_{n}$.

- 2nd case: If $x_{0} \in \bar{\Omega} \backslash(K \cup \mathcal{S})$ then $\rho(x, p) \geq 1$ if and only if $H(x, p) \geq 0$ and singular and discontinuous viscosity supersolution coincide. Hence the previous argument can be adapted to show that $u$ is a viscosity supersolution also in this case.

\section{Applications of the stability to the Shape-From-SHading}

In SFS, it is very important to design schemes and algorithms robust respect to noise and errors on parameters that the user must input, for example the focal length, the size of the pixels (width, height), the position and the direction of the light source (following the chosen modeling).

Mathematically, the robustness is expressed by the continuity of the application which from an image $I$ (a focal length $f$ or a light source direction $\mathbf{L}, \ldots$, respectively), returns the solution $u$ of the associated PDE. If we denote $H_{n}$ the Hamiltonian obtained by replacing the parameters $\mathbf{L}, f$ and $I$ by $\mathbf{L}_{n}, \mathrm{f}_{n}$ and $I_{n}$ in $H$, then the desired stability property corresponds to the convergence of the SDVSs of (4.1)-(2.7) towards the SDVS of (2.1)-(2.7) when $n \rightarrow+\infty$. Here we analyze the robustness of SDVS with respect to perturbations of the image brightness $I$ (for perturbations of $\mathbf{L}$ and $\mathbf{f}$ and stability of approximation schemes we refer to the companion paper [24]).

\subsection{Approximation of the degenerated equations by non-degenerated equations}

The lack of uniqueness of the solution to (2.1) is a noteworthy problem for numerical computations of a solution to the shape-from-shading problem, since it causes numerical instability and sometimes fail of convergence of standard approximation schemes. It is therefore usual to regularize (2.1) by cutting the image brightness at a certain level strictly less than 1 before applying the approximation procedure. As a first application of Theorem 4.3, we show that the notion of SDVS is stable with respect to this type of regularization.

Given a continuous image $I$ and $n$, we set

$$
I_{n}(x)=\min \left\{I(x), 1-\frac{1}{n}\right\}, \quad x \in \bar{\Omega}
$$


For a SFS Hamiltonian $H$, we denote by $H_{n}$ the new Hamiltonian obtained replacing $I(x)$ by $I_{n}(x)$ in $H$. Since $I_{n} \leq I$, the reader will verify easily that for all the SFS Hamiltonians,

$$
H_{n}(x, p) \leq H(x, p) \quad \text { for any } x \in \bar{\Omega}, p \in \mathbb{R}^{N} .
$$

Therefore,

$$
\mathcal{Z}(x) \subset \mathcal{Z}_{n}(x) \quad x \in \bar{\Omega} .
$$

So the condition (4.3) holds for $\theta_{n}=1$. Moreover, it is easy to prove that $H_{n}$ converge toward $H$ (when $n \rightarrow \infty)$ uniformly with respect to $(x, p) \in \bar{\Omega} \times B(0, R)$ for any $R>0$. Therefore, the singular viscosity solutions of (4.1)-(2.7) converge toward the unique singular viscosity solution of (2.1)-(2.7).

Now, let us remark that, for any $n$, the SFS Hamiltonian $H_{n}$ (associated with $I_{n}$ ) is not degenerate anymore (i.e. $\mathcal{S}_{n}=\emptyset$ ). So, its (unique) singular viscosity solution is the (unique) discontinuous viscosity solution. Thus, for approximating its solution, we can use the classical tools we have developed in [22].

\subsection{Robustness of the shape-from-shading solutions to image regularization and pixel noise}

In computer vision or more generally in image processing, the images are always corrupted by noise. To remove this noise, the images are often regularized [29]. In other respects, most of CCD sensors slightly smooth the images and defocus effects can strongly diffuse the brightness information. Since, we do not have taken into account these regularization effects in the modeling, it seems important to guarantee the robustness of our SFS methods with respect to them.

If we consider a sequence of noisy (or denoised) images $I_{n}$ converging uniformly to $I$, unfortunately the stability in general does not hold. It is possible to design counter-examples for which $I_{n}$ converges uniformly toward $I$ but the corresponding SDVSs do not converge uniformly (see for example [5]). Here we show that if the images $I_{n}$ are appropriately regularized, we recover again the stability of SDVSs.

Set $\omega_{n}=\left\|I-I_{n}\right\|_{L^{\infty}(\Omega)}$ and let $\varepsilon_{n}$ be a sequence such that $\omega_{n} / \varepsilon_{n} \rightarrow 0$ for $n \rightarrow+\infty$. Set

$$
I_{n \varepsilon_{n}}=\min \left(I_{n}(x), 1-\varepsilon_{n}\right), \quad x \in \bar{\Omega},
$$

let $H_{n}(x, p)$ be the SFS Hamiltonians corresponding to the image brightness $I_{n \varepsilon_{n}}$ and $\mathcal{Z}_{n}=\left\{p \in \mathbb{R}^{N}: H_{n}(x, p) \leq\right.$ $0\}$. For simplicity we assume that both the limit equation (2.1) and the regularized equations corresponding to the Hamiltonians $H_{n}$ admit $\psi \equiv 0$ as a subsolution ${ }^{1}$. We want to show that assumption (4.3), with $\psi \equiv 0$, holds (the other assumptions of the stability theorem being obvious).

Set $\mathcal{D}^{n}=\left\{x \in \Omega: I(x) \geq 1-\varepsilon_{n}\right\}$. We distinguish two cases:

- If $x \in \mathcal{D}^{n}$, then $I_{n \varepsilon_{n}}(x) \leq 1-\varepsilon_{n} \leq I(x)$, hence, recalling that the SFS Hamiltonians are increasing in $I$, we get $\mathcal{Z}(x) \subset \mathcal{Z}_{n}(x)$.

- If $x \notin \mathcal{D}^{n}$, then

$$
\begin{aligned}
I(x) \geq I_{n}(x)-\omega_{n} & \geq I_{n \varepsilon_{n}}(x)-\omega_{n}=I_{n \varepsilon_{n}}(x)-\frac{\omega_{n}}{\varepsilon_{n}} \varepsilon_{n} \\
& \geq I_{n \varepsilon_{n}}(x)-\frac{\omega_{n}}{\varepsilon_{n}}\left(I_{n \varepsilon_{n}}(x)-1\right) \geq\left(1-\frac{\omega_{n}}{\varepsilon_{n}}\right) I_{n \varepsilon_{n}}(x) .
\end{aligned}
$$

Therefore, recalling that $\omega_{n} / \varepsilon_{n} \rightarrow 0$, we find that hypothesis (4.3) is satisfied with $\theta_{n}=\left(1-\frac{\omega_{n}}{\varepsilon_{n}}\right)^{-1}$.

Example. A typical example of a denoised sequence of images is given by $I_{n}(x)=\left(I * \eta_{n}\right)(x)$, where $\eta_{n}$ is a standard mollifier, i.e. $\eta_{n}(x)=n^{N} \eta(n x)$ with $\eta: \mathbb{R}^{N} \rightarrow \mathbb{R}$ a smooth, nonnegative function such that the support of $\eta$ is contained in the unit ball and $\int_{\mathbb{R}^{N}} \eta(z) \mathrm{d} z=1$ (we assume for simplicity that $I$ is defined in a

\footnotetext{
${ }^{1}$ Let us recall that by an appropriate change of variables, the SFS Hamiltonians $H_{P / F}^{p e r s}$ and $H_{R / T}^{\text {orth }}$ can be reduced to this case.
} 
neighborhood of $\bar{\Omega}$, so $I_{n}$ can be defined in $\bar{\Omega}$ for $n$ sufficiently large). $I_{n}$ is a smooth function and $0 \leq I_{n}(x) \leq 1$. Moreover $I_{n}(x)=1$ if and only if $I(y)=1$ for any $y \in B(x, 1 / n)$. Hence $\mathcal{S}_{n}=\left\{x \in \Omega: I_{n}=1\right\}$ is a proper subset of $\mathcal{S}$. If $\mathcal{S}$ reduces for example to a finite number of points, the regularized problem is not singular. Note that $H_{n}$ satisfies the same hypothesis of $H$, i.e. it is continuous, convex and, since $I \geq m>0$ implies $I_{n} \geq m>0$, also coercive in $p$.

\section{A GenerAl FRAMEWORK FOR SFS}

We now show that the notion of SDVS provides a general mathematical framework unifying the previous theory in the SFS literature based on viscosity solutions. In this section, since we are interested in fixing the values of the solution in a subset of the singular set, we assume that $K \subset \mathcal{S}$.

For a given function $u$, we denote by $\Pi_{u}$ the set of points in $\Omega$ such that a constant function is $S$-subtangent to $u-\psi$ at $x$. In other words $\Pi_{u}$ is the set of minimum points of $u-\psi$. We also set

$$
\Gamma_{u}=\left\{x \in K \mid \exists y \in B_{S}(x) \text { verifying } u(x) \geq \varphi(y)+\psi(x)-\psi(y)\right\}
$$

(recall Def. 2.13).

Theorem 6.1. Let $u$ be a (discontinuous) viscosity solution of (2.1)-(2.7) such that $u(x) \leq \varphi(x)$ for any $x \in K$. Then $u$ is the SDVS of (2.1)-(2.7) if and only if $\Pi_{u} \subset \Gamma_{u}$.

Proof. First observe that a SDVS cannot have points of local minimum in $\Omega$ outside of $\Gamma_{u}$. In effect, by contradiction, if $u-\psi$ admits a constant function $S$-subtangent to $x_{0} \notin \Gamma_{u}$, then the function $\psi$ is a $S$-subtangent to $u$ at $x_{0}$. Since by the definition of $\mathcal{S}, \psi$ is a strict subsolution of (2.1) it follows that $u$ cannot be a (singular) supersolution at $x_{0}$.

To prove the other assertion, we have just to prove that a (discontinuous) viscosity solution $u$ is a singular supersolution at all the singular points which are not in $\Gamma_{u}$. We assume for simplicity that $B_{S}\left(x_{0}\right)=\left\{x_{0}\right\}$ but it is straightforward to extend the argument to the general case.

We argue by contradiction assuming that there exists a function $\phi$, a neighborhood $A \subset \Omega \backslash \Gamma_{u}$ of $x_{0}$ and $\theta \in] 0,1\left[\right.$ such that $\phi$ is a $S$-subtangent to $u$ at $x_{0}$ with $\phi\left(x_{0}\right)=u\left(x_{0}\right), \phi(x) \leq u(x)$ for $x \in A$,

$$
\phi(x) \leq u(x)-\eta, \quad x \in \partial A
$$

for some $\eta>0$ and

$$
\rho(x, \nabla \phi) \leq \theta \quad x \in A
$$

in the viscosity sense. Since $u$ is a solution of (2.1) in $A$ we have (see $[1,17]$ )

$$
u(x)=\psi(x)+\min \left\{u(y)-\psi(y)+L_{A}(x, y): y \in \partial A\right\} \wedge\left\{u\left(x_{0}\right)-\psi\left(x_{0}\right)+L_{A}\left(x, x_{0}\right)\right\} .
$$

Since $x_{0}$ is not a minimum point of $u-\psi$, we can find $x_{n}$ such that $L\left(x_{n}, x_{0}\right) \rightarrow 0$ for $n \rightarrow \infty$ and $u\left(x_{n}\right)-\psi\left(x_{n}\right) \leq$ $u\left(x_{0}\right)-\psi\left(x_{0}\right)$. It follows that $u\left(x_{0}\right)-\psi\left(x_{0}\right)+L_{A}\left(x_{n}, x_{0}\right)>u\left(x_{n}\right)-\psi\left(x_{n}\right)$ (since $\left.L_{A}\left(x_{n}, x_{0}\right)>0\right)$. By (6.1), we can find $y_{n} \in \partial A$ such that $u\left(x_{n}\right)=\psi\left(x_{n}\right)+u\left(y_{n}\right)-\psi\left(y_{n}\right)+L_{A}\left(x_{n}, y_{n}\right)$. Hence

$$
\begin{aligned}
0 & \geq u\left(y_{n}\right)-\psi\left(y_{n}\right)-u\left(x_{0}\right)+\psi\left(x_{0}\right)+L_{A}\left(x_{n}, y_{n}\right)-L_{A}\left(x_{n}, x_{0}\right) \\
& \geq \phi\left(y_{n}\right)+\eta-\psi\left(y_{n}\right)-\phi\left(x_{0}\right)+\psi\left(x_{0}\right)+L_{A}\left(x_{n}, y_{n}\right)-L_{A}\left(x_{n}, x_{0}\right) .
\end{aligned}
$$

Passing to a subsequence we can assume that $y_{n} \rightarrow y_{0}$ with $y_{0} \in \partial A$. Since $L_{A}\left(x_{n}, y_{n}\right)$ and $L_{A}\left(x_{0}, y_{n}\right)$ converge to $L_{A}\left(x_{0}, y_{0}\right)$ and $L_{A}\left(x_{n}, x_{0}\right)$ converges to 0 , we can find $n$ large such that

$$
L_{A}\left(x_{n}, y_{n}\right)-L_{A}\left(x_{n}, x_{0}\right)+\eta>L_{A}\left(x_{0}, y_{n}\right) .
$$


Substituting the previous inequality in (6.2) we get

$$
0>\phi\left(y_{n}\right)-\psi\left(y_{n}\right)-\phi\left(x_{0}\right)+\psi\left(x_{0}\right)+L_{A}\left(x_{0}, y_{n}\right) .
$$

Fixed such $n$, we can find $\xi \in W^{1, \infty}([0,1], \bar{A})$ joining $x_{0}$ to $y_{n}$ satisfying

$$
\int_{0}^{1}\left(\delta(\xi(t),-\dot{\xi}(t))+\frac{\mathrm{d}}{\mathrm{d} t}(\phi(\xi(t))-\psi(\xi(t)))\right) \mathrm{d} t<0 .
$$

From the previous inequality we get a contradiction to the definition of singular viscosity supersolution as in the proof of Theorem 3.4.

The previous theorem says, in other words, that the SDVS is the unique (discontinuous) viscosity solution $u$ of (2.1)-(2.7) verifying $u(x) \leq \varphi(x)$ for any $x \in K$ without local minima on $\Omega \backslash \Gamma_{u}$. An interesting interpretation of Theorem 6.1 is the following (recall that we are assuming $K \subset \mathcal{S}$ ):

Corollary 6.2. The (discontinuous) viscosity solutions of (2.1)-(2.7) can be characterized only by their minima, i.e. if $u$ is a (discontinuous) viscosity solutions of (2.1)-(2.7) then $u$ is the (unique) SDVS of

$$
\begin{cases}H(x, \nabla u)=0, \quad \forall x \in \Omega, \\ u(x)=\hat{\varphi}(x), \quad \forall x \in \partial \Omega \cup \mathcal{S}\end{cases}
$$

where $\hat{\varphi}(x)=\varphi(x)$ for any $x \in \Pi_{u} \cup \partial \Omega$ and $\hat{\varphi}(x)=+\infty$ if $x \in \mathcal{S} \backslash \Pi_{u}$.

Thus this result extends the work of Dupuis and Oliensis [11]. In [11], Dupuis and Oliensis characterize the $C^{1}$ solutions by their values at the local minimum points ${ }^{2}$. Here, we have extended this characterization to the (discontinuous) viscosity solutions. Finally, let us emphasize that the notion of SDVS allows to unify the various theories based on viscosity solutions used for solving the SFS problem. In effect,

- in the case where the Dirichlet boundary conditions (DBC) are finite on $\partial \Omega \cup \mathcal{S}$ and the compatibility condition (see [17]) holds, then the SDVS of (2.1)-(2.7) is the continuous viscosity solution used by $[18,21,23,25]$;

- in the case where the DBC are finite on $\partial \Omega$ and where there do not exist singular points, then the SDVS of (2.1)-(2.7) coincides with the discontinuous viscosity solution used by [21,22] (the compatibility conditions are no more required);

- when the DBC are finite on the boundary of the image and all the singular points are "sent to infinity", the SDVS of (2.1)-(2.7) corresponds to the Camilli and Siconolfi's singular viscosity solutions [6,7] used by Falcone and Sagona [12];

- as we have demonstrated above the SDVSs coincide with the $C^{1}$ solutions of (2.1) verifying the Assumption 2.1 in [11] (when smooth solutions exist). Therefore, when there do not exist $C^{1}$ solutions, the notion of SDVS allows to extend the work of Dupuis and Oliensis [11].

\section{EXAMPles OF NUMERICAL RESUltS}

In the companion paper [24], we explain how to compute a numerical approximation of the SDVS of the generic SFS equation. This requires three steps. First, we regularize the equation. Second, we approximate the regularized SFS equation by approximation schemes. Finally, from the approximation schemes, we design numerical algorithms. The convergence of the computed solutions towards the SDVS is proved. The numerical results displayed here are computed by using the algorithm presented in [24].

\footnotetext{
${ }^{2}$ Let us recall that in [11], the cost function $l$ has to be positive. For this reason Dupuis and Oliensis need to introduce the SFS Hamiltonian $H_{D / O}^{\text {orth }}\left(\right.$ instead of dealing with $H_{R / T}^{\text {orth }}$ ). Here, we relax this constrained assumption.
} 


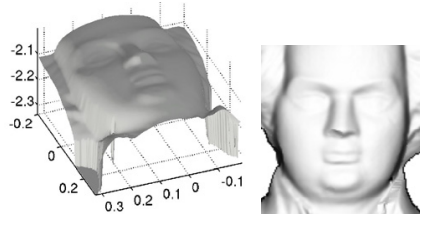

a)

b)

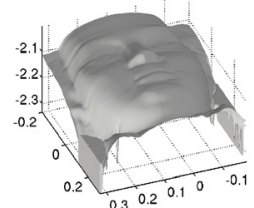

c)

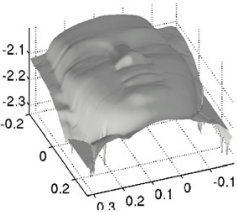

d)

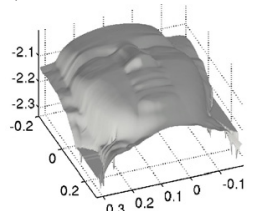

e)

Figure 1. Reconstruction of Mozart's face with and without boundary data. a) original surface; b) image generated from a); c) SDVS of the Perspective SFS equation [21] associated to the image b) complemented with the exact boundary data on the boundary of the image and at all critical points $(\varphi(x)=+\infty$, elsewhere); d) SDVS of the same equation as c) complemented with the exact data (only) at the "minimal" critical points $(\varphi(x)=+\infty$ elsewhere; in particular we impose state constraints on the boundary of the image); e) SDVS of the same equation as c) with $\varphi(x)=+\infty$ everywhere except at the critical point on the nose.

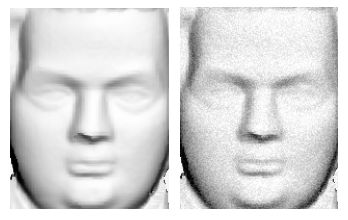

a)

b)

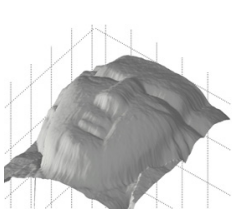

c)

FIGURE 2. Stability of the SDVS with respect to wrong parameters and pixel noise. a) Image generated from Mozart's face represented in Figure 1a with $\mathbf{l}=(0.1,-0.3)$ and $\mathrm{f}=3.5 ; \mathrm{b})$ noisy image $(\mathrm{SNR} \simeq 5)$; c) SDVS reconstructed from b) by using the incorrect parameters $\mathbf{l}_{\varepsilon}=(0.2,-0.1)$ and $\mathbf{f}_{\varepsilon}=10.5$, and with $\varphi(x)=+\infty$ everywhere except at the critical point on the nose.

In the examples above, we deal with the classical Mozart's face [30]. Figure 1 shows the SDVS reconstructed from the Mozart's face image associated with the exact boundary data on the boundary of the image and at all critical points (Fig. 1c), associated with the exact boundary data (only) at all the "minimal" critical points (Fig. 1d), and associated with the exact boundary data only at the critical point on the tip of the nose (Fig. 1e) (in the three cases, we impose $\varphi(x)=+\infty$ at all the other points $x$ ). Finally, the stability of the SDVS can be illustrated by Figure 2. This figure displays the SDVS computed from the Mozart's face image perturbed by additive uniformly distributed white noise $(\mathrm{SNR} \simeq 5)$ with wrong focal length and light parameters and without any boundary data $(\varphi(x)=+\infty$ for all $x$ except at the critical point on the nose). The angle between the initial light vector $\mathbf{L}$ and the corrupted light vector $\mathbf{L}_{\varepsilon}$ is around $13^{\circ}$. The focal length error is around 200\%. More numerical results can be found in [24].

\section{REFERENCES}

[1] M. Bardi and I. Capuzzo-Dolcetta, Optimal control and viscosity solutions of Hamilton-Jacobi-Bellman equations. Birkhauser, Boston (1997).

[2] G. Barles, An approach of deterministic control problems with unbounded data. Ann. I. H. Poincaré 7 (1990) 235-258. 
[3] G. Barles, Solutions de Viscosité des Equations de Hamilton-Jacobi. Springer-Verlag, Paris (1994).

[4] G. Barles and B. Perthame, Comparison principle for Dirichlet-type Hamilton-Jacobi equations and singular perturbations of degenerated elliptic equations. Appl. Math. Opt. 21 (1990) 21-44.

[5] I. Barnes and K. Zhang, Instability of the eikonal equation and shape-from-shading. ESAIM: M2AN 34 (2000) $127-138$.

[6] F. Camilli and A. Siconolfi, Maximal subsolutions for a class of degenerate Hamilton-Jacobi problems. Indiana U. Math. J. 48 (1999) 1111-1132.

[7] F. Camilli and A. Siconolfi, Nonconvex degenerate Hamilton-Jacobi equations. Math. Z. 242 (2002) 1-21.

[8] I. Capuzzo-Dolcetta and P.-L. Lions, Hamilton-Jacobi equations with state constraints. Trans. Amer. Math. Soc. 318 (1990) $643-68$.

[9] F.H. Clarke, Optimization and Nonsmooth Analysis. SIAM, Classics in Applied Mathematics 5, Philadelphia (1990).

[10] M.G. Crandall and P.-L. Lions, Viscosity solutions of Hamilton-Jacobi equations. Trans. Amer. Math. Soc. 277 (1983) 1-42.

[11] P. Dupuis and J. Oliensis, An optimal control formulation and related numerical methods for a problem in shape reconstruction. Ann. Appl. Probab. 4 (1994) 287-346.

[12] M. Falcone and M. Sagona, An algorithm for the global solution of the Shape-From-Shading model, in Proceedings of the International Conference on Image Analysis and Processing. Lect. Notes Math. 1310 (1997) 596-603.

[13] B.K. Horn and M.J. Brooks, Eds., Shape From Shading. The MIT Press (1989).

[14] H. Ishii, A boundary value problem of the Dirichlet type for Hamilton-Jacobi equations. Ann. Scuola Norm. Sup. Pisa Cl. Sci. 16 (1989) 105-135.

[15] H. Ishii and M. Ramaswamy, Uniqueness results for a class of Hamilton-Jacobi equations with singular coefficients. Commun. Part. Diff. Eq. 20 (1995) 2187-2213.

[16] R. Kimmel, K. Siddiqi, B.B. Kimia and A. Bruckstein, Shape from shading: Level set propagation and viscosity solutions. Int. J. Comput. Vision 16 (1995) 107-133.

[17] P.-L. Lions, Generalized Solutions of Hamilton-Jacobi Equations. Res. Notes Math. 69. Pitman Advanced Publishing Program, London (1982).

[18] P.-L. Lions, E. Rouy and A. Tourin, Shape-from-shading, viscosity solutions and edges. Numer. Math. 64 (1993) 323-353.

[19] M. Malisoff, Bounded-from-below solutions of the Hamilton-Jacobi equation for optimal control problems with exit times: vanishing Lagrangians, eikonal equations, and shape-from-shading. NoDEA: Nonlinear Differ. Equ. Appl. 11 (2004) 95-122.

[20] J. Oliensis and P. Dupuis, Direct method for reconstructing shape from shading, in Proceedings of SPIE Conf. 1570 on Geometric Methods in Computer Vision (1991) 116-128.

[21] E. Prados and O. Faugeras, Perspective shape-from-shading, and viscosity solutions, in Proceedings of the 9th International Conference on Computer Vision (Nice 2003). IEEE Comput. Soc. Press 2 (2003) 826-831.

[22] E. Prados and O. Faugeras, A generic and provably convergent shape-from-shading method for orthographic and pinhole cameras. Int. J. Comput. Vision 65 (2005) 97-125.

[23] E. Prados, O. Faugeras and E. Rouy, Shape from shading and viscosity solutions, in Proceedings of the 7th European Conference on Computer Vision (Copenhagen 2002), Springer-Verlag 2351 (2002) 790-804.

[24] E. Prados, F. Camilli and O. Faugeras, A unifying and rigorous shape from shading method adapted to realistic data and applications. J. Math. Imaging Vis. (2006) (to appear).

[25] E. Rouy and A. Tourin, A viscosity solutions approach to shape-from-shading. SIAM J. Numer. Anal. 29 (1992) $867-884$.

[26] H.M. Soner, Optimal control with state space constraints. SIAM J. Control Optim 24 (1986): Part I: 552-562, Part II: $1110-1122$.

[27] H.J. Sussmann, Uniqueness results for the value function via direct trajectory-construction methods, in Proceedings of the 42nd IEEE Conference on Decision and Control 4 (2003) 3293-3298.

[28] A. Tankus, N. Sochen and Y. Yeshurun, A new perspective [on] Shape-From-Shading, in Proceedings of the 9th International Conference on Computer Vision (Nice 2003). IEEE Comput. Soc. Press 2 (2003) 862-869.

[29] D. Tschumperlé, PDE's Based Regularization of Multivalued Images and Applications. Ph.D. Thesis, University of Nice-Sophia Antipolis (2002).

[30] R. Zhang, P.-S. Tsai, J.-E. Cryer and M. Shah, Shape from shading: A survey. IEEE T. Pattern Anal. 21 (1999) 690-706. 